\title{
Clinical efficacy of anal fistula plug treatment regimens in anal fistula patients.
}

\author{
Xiaojie Fang ${ }^{1}$, Chenyun Miao ${ }^{2}$, Yexiao Hu${ }^{1}$, Chun Jin $^{1}$, Wanle Hu${ }^{1}$, Zhao Jin ${ }^{3}$, Linmin Zhuge \\ Chenguo Zheng ${ }^{*}$
}

${ }^{1}$ Department of Anorectal surgery, The Second Affiliated Hospital and Yuying Children's Hospital of Wenzhou Medical University, No. 109, West College Road, Lucheng District, Wenzhou, PR China

${ }^{2}$ Department of Gynecology (TCM), Wenzhou Traditional Chinese Medical Hospital, Wenzhou, PR China

${ }^{3}$ Department of Anorectal Surgery, Wenzhou City Hospital of Traditional Chinese Medicine and Western Medicine Combined, Wenzhou, PR China

\begin{abstract}
Aim: Anal Fistula Plug (AFP) is considered as a new promising technique in treating anal fistula patients, mainly due to it could maintain the integrity of patients' integrity and decrease patients' discomfort with a simple operation. It also is beneficial to the following surgical options if needed. However, the underlying clinical efficacy is still unknown. Herein, we aimed to investigate the clinical efficacy of AFP in anal fistula patients by a retrospective analysis.

Methods: We collected 52 anal fistula patients from the $2^{\text {nd }}$ affiliated hospital of Wenzhou Medical University and the hospital of traditional Chinese and Western medicine in Wenzhou between August 2012 and July 2016. Among them, 25 patients were treated with AFP, while 27 patients were treated with incision-thread-drawing. After treatments, all patients were evaluated by analyzing the differences on postoperative pain, cure rates, healing time, scar area and anal sphincter function at the subsequent follow-up.

Results: The results showed that there were significant differences on postoperative pain between the two groups $(\mathbf{P}<0.001)$. Further, the healing time in patients with AFP treatment was remarkably lower than those with incision-thread-drawing treatment $(\mathbf{P}<\mathbf{0 . 0 5})$, whereas the anal scar area and the functional score of anal sphincterin patients with AFP treatment was markedly higher than those with incision-thread-drawing treatment $(\mathbf{P}<\mathbf{0 . 0 0 1})$. Nevertheless, the cure rates were not obviously different between the two groups.

Conclusions: Taken together, these data suggested that AFP procedure could notably alleviate postoperative pain, shorten healing time, and retain anal sphincter function as compared to incisionthread-drawing procedure.
\end{abstract}

Keywords: Anal fistula plug, Clinical efficacy, Retrospective analysis.

Accepted on November 14, 2017

\section{Introduction}

Anal fistula remains one of the most common anorectal diseases with an incidence of 8.6 per 100,000 patients/year [1]. Although traditional therapeutic options, such as fistulectomy, thread-drawing therapy, fibrin glue application etc., were used for the management of anal fistula with good healing rates, the clinical efficacy of these operations was not entirely satisfactory primarily due to long healing time, large size wounds and impaired anal function which seriously affect life quality of patients [2,3]. Moreover, high recurrence rate and high incontinence rate followed by the above operations are still enormous challenges, even for experienced anorectal surgeons [4,5]. Currently, according to the latest anal fistula treatment guidelines of American Society of Colon and Rectal Surgeons (ASCRS), it clearly pointed that it was equally important for treated patients in healing anal fistula and maintaining anal function, meanwhile it also must be balanced among the degree of sphincter incision, cure rate and postoperative anal function [6]. Therefore, the ideal therapeutic goal for anal fistula should aim to minimize or eradicate the injury to the anal sphincters, promote healing of the tract, preserving the sphincters' function and prevent future recurrence $[1,5]$.

Anal Fistula Plugs (AFPs), derived from porcine small intestinal submucosa, is an absorbable biomaterial, which could provide a scaffold for host fibroblasts to promote tissue 
healing and repair damaged tissue [7,8]. AFP was first described in 2006 by Johnson et al to treat anal fistula in the attempt to achieve closure. Furthermore, Johnson et al also compared the differences of AFP and fibrin glue (another biological infill material) in clinical efficacy and discovered that the healing rates were $87 \%$ and $40 \%$ in anal fistula patients with AFP and fibrin glue treatments, respectively [9]. Hence, AFP was regarded as a good therapeutic option for anal fistula patients [10]. It changed the therapeutic pattern from traditional cutting surgeries to invasive conservative methods [4]. Even though many surgeons have been turning to the use of AFP to occlude the fistula tract, support tissue remodelling and promote healing, there has been no systematic assessment between AFP procedure and traditional incision-threaddrawing procedure on the clinical efficacy. Thus, the present study aimed to determine the potential curative effects of AFP on the treatment of anal fistula patients by analyzing clinical related examination indicators, including postoperative pain, cure rates, healing time, scar area and anal sphincter function, in comparison with a traditional incision-thread-drawing method.

\section{Materials and Methods}

\section{Patients' information}

This trial was conducted as a retrospective study in the $2^{\text {nd }}$ affiliated hospital of Wenzhou Medical University and the hospital of traditional Chinese and Western medicine in Wenzhou. In total, 52 anal fistula patients with other anorectal diseases background were enrolled in the study from August 2012 to July 2016. This study was performed after the approval of the Medical Research Ethics Committee with written informed consents obtained from all participants included in the study. Additionally, all patients would make a further definite diagnosis in the following operation.

Among these patients, 47 patients were male gender $(90.4 \%)$ and 5 patients were female gender $(9.6 \%)$. Moreover, the median age of these patients was $37.04 \mathrm{y}$. Twenty-five patients, including 22 males and 3 females (the median age was 36.56 years, ranged 23 65 y), underwent AFP operation, while twenty-seven patients, including 25 males and 2 females (the median age was $37.48 \mathrm{y}$, ranged 13 64 y), underwent incisionthread-drawing operation.

\section{Diagnostic criteria}

To date, the approaches to accurate diagnosis in anal fistula are still no established "golden standard". Therefore, according to recent relevant literatures and personal experience, we formulated the diagnostic criteria below.

Low anal fistula: Low anal fistula was defined as that with the fistula tract crossing the under part of deep external sphincter. Also, it is divided into two types, namely low simple anal fistula and low complexity anal fistula. (1) Low simple anal fistula only has one fistula with an internal opening in the anus fossae. (2) Low complexity anal fistula has two or more external orifice and two or more fistula connected to the inlet.

High anal fistula: High anal fistula was defined as that with the fistula tract crossing the upper part of deep external sphincter and invading puborectal muscle or elevator ani muscle. Similarly, it is also divided into two types, namely high simple anal fistula and high complexity anal fistula. (1) High simple anal fistula only has one fistula with an internal opening in the anus fossae. (2) High complexity anal fistula has two or more external orifice and at least two fistulas connected to the inlet or a branch pipe cavity.

\section{Inclusion/exclusion criteria}

Inclusion criteria: (1) one fistula with an internal opening in the anal crypt; (2) one or more fistula with an external opening; (3) the direction of fistula and the position relationship between the anorectal and the internal opening of fistula detected by the examinations of rectum $\mathrm{B}$ ultrasound, probe checking or fistula imaging.

Exclusion criteria: (1) with cardiovascular, hematopoietic system, liver, kidney and other serious diseases; (2) perianal dermatosis such as eczema and diarrhea; (3) tuberculosis, Crohn's disease caused by the specific anal fistula.

\section{Surgical procedure}

The bowel areas of all patients were prepared by enema cleaning with sodium phosphate rectal solution at the night before the surgery or the morning of before the surgery. Then, all patients received broad spectrum antibiotics (i.e., cefoxitin sodium) at half an hour before the surgery. Additionally, based on the anal fistula types, we accordingly chose different spinal anesthesia or sacral anesthesia, lithotomy position or left lateral decubitus position. And all surgical procedures were performed by two accredited anorectal surgeons.

For AFP operation patients, surgeons firstly adopted fistula brush and hydrogen peroxide instillation to clean fistula and identify the location of anal fistula. Meanwhile, the tract was not curetted to avoid enlarging or damaging the fistula tract. Then, the fistula brush was inserted in the fistula track and AFP device (C-AFP-0.6 $\times$ 9.5, Biodesign ${ }^{\mathrm{TM}}$ Surgisis), which was submerged in $0.9 \%$ saline for $1 \sim 2 \mathrm{~min}$ before use, was implanted into the internal opening of fistula track by connecting the internal end of fistula brush until resistance was felt and then fixed in this place with vicryl 2-0 stitche. Afterwards, the external orifice of the fistula was not completely occluded so that the track could drain and excess AFP materials that remained outside the external opening of the fistula were removed. Finally, the operations were considered successful if the fistula was not obviously infected and drainage was totally stopped at least $6 \sim 12 \mathrm{~W}$.

For incision-thread-drawing operation patients, surgeons firstly used a probe or methylene blue to determine the entire fistula tract's position. Then, care was taken to ensure that a long fusiform incision along the external orifice lesion tissue was 
made for drainage, the inflammatory tissues, necrotic tissues and scars of fistula were remove with a curet, and the fistula was also excised until anal sphincter verge at the level of the external opening was encountered. A probe was placed in the external orifice of fistula and gently withdrawn through the fistula. Thereafter, a rubber band was inserted into the probe using blood vessel forceps clamps and tightly tied on the probe with silk thread to maintain a constant tension. Finally, these materials were fixed on crissumm, meanwhile wounds were cleaned using methylene blue+lidocaine solution and filled with Vaseline gauze. In addition, sterile gauze was externally applied to fix the wounds.

After the surgery, all patients were instructed to stay on a semi liquid diet and not allowed to defecate at least $48 \mathrm{~h}$. In order to prevent the wounds from infections, the broad spectrum antibiotics (i.e., cefoxitin sodium) were administrated to all patients for three days by intravenous drip. Moreover, after defecating, the wounds should be cleaned and dressed daily to further avoid anal contraction. Meanwhile, patients were advised to refrain from physical labour and strenuous sports for $2 \mathrm{w}$ following the operation to prevent anal fistula fall off.

\section{Postoperative period and follow up}

Follow-up visit was performed by an independent observer by means of a telephone interview and physical re-examination and scheduled once a week after one month when all patients were discharged. Then, the follow-up visit was planned every other month and ultimately on an individual basis as clinically appropriate. The primary endpoint of this study was a successful healing defined as closure of the external fistula opening with no drainage, infection, perianal swelling or abscess formation at 3 months postoperatively for AFP and incision-thread-drawing groups. Secondary endpoints included recurrence rate, postoperative pain, postoperative anal function and postoperative scar area.

\section{Postoperative evaluation criteria}

Curative standard: In accordance with the anal fistula curative standards made by National Anorectal Conference, the therapeutic effect was totally divided into two categories: (1) Recovered: The wounds were completely healed with absence of the clinical symptoms. (2) Unrecovered: The wounds were not successfully healed with fistula drainage and the clinical symptoms were not improved.

Pain degree evaluation: Numeric Rating Scales (NRS) are used to evaluate postoperative pain, including 10 degrees which was corresponding divided into 1 10 scores.

Anus function assessment: Anal function alterations were assessed by the International Wexner Continence Grading Scale, ranged from 0 (normal continence) to 20 (maximum incontinence with maximum disturbance of lifestyle).

\section{Statistical analysis}

All data were expressed as (mean+standard deviation) or (median+range). The chi-square test or Fisher's exact test is used to compare the difference between the groups, while t-test or the Mann-Whitney $U$ tests were used to compare the continuous variables between the groups. Additionally, repetitive measure variance analysis was used to estimate the changes of anal function by NRS scores from baseline to $30 \mathrm{~d}$. Eventually, statistical analysis was performed by using SPSS, version 21.0 and statistically significant differences were defined by a $P$ value less than 0.05 .

\section{Results}

\section{Clinical diagnostic of patients}

The study started recruiting patients from August 2012 to July 2016. According to the diagnostic criteria mentioned above, surgeons decided the types of these anal fistula patients by identifying the position of anal fistula and calculating the number of external orifice and fistula. Subsequently, the 52 anal fistula patients were randomly assigned into two groups. The basic information of these chosen patients was shown in Table 1. There were no notably differences on the length of fistula $(\mathrm{t}=-0.79, \mathrm{P}=0.937)$, the types of fistula $\left(\chi^{2}=4.746\right.$, $\mathrm{P}=0.191)$ and the number of external orifice $\left(\chi^{2}=2.831\right.$, $\mathrm{P}=0.243$ ) between the two groups, thereby these factors didn't affect the following results. However, the position of external fistula and number of fistula in AFP group were remarkably different from that in incision-thread-drawing group $\left(\chi^{2}=7.597\right.$, $\mathrm{P}=0.022$ ).

\section{Postoperative assessment}

The detailed results about all indicators of postoperative assessment were displayed in Table 2.

\section{Healing rate and time}

During the follow-up period, we found that 6 patients, including 4 low simple anal fistulas and 2 high complex anal fistulas, were not healed in AFP group, while 2 high complex anal fistula patients were not healed in incision-thread-drawing group, suggesting the number of failure operations and the challenge of high complexity anal fistula in the two groups. However, the healing rates were $76 \%$ (19/25 represented 19 patients healed and 25 patients involved in AFP treatments) and $93 \%$ (25/27 represented 25 patients healed and 27 patients involved in incision-thread-drawing treatments) in AFP and incision-thread-drawing groups, respectively. And there were no significant differences in healing rate between the two groups $\left(\chi^{2}=2.745, \mathrm{P}=0.098\right)$.

In addition, the length of healing was $(31.96 \pm 2.54 \mathrm{~d})$ in AFP group, whereas that was $(39.44 \pm 12.42 \mathrm{~d})$ in incision-threaddrawing group. Moreover, the medium healing time was $33 \mathrm{~d}$ (rang 26 36 d) and $40 \mathrm{~d}$ (rang 26 36 d) in AFP and incisionthread-drawing groups, respectively. By t-test analysis, we discovered that there were a remarkably difference in the 
length of healing between the two groups $(\mathrm{t}=-3.06, \mathrm{P}=0.005)$, indicating that the recovery speed in AFP group was faster than that in incision-thread-drawing group.

\section{Scar area}

The results exhibited a statistical difference $(\mathrm{t}=-8.74, \mathrm{P}<0.001)$ that the scar area was $\left(0.33 \pm 0.12 \mathrm{~cm}^{2}\right)$ in AFP group, but the scar area was $\left(3.06 \pm 1.62 \mathrm{~cm}^{2}\right)$ in incision-thread-drawing group. Therefore, these data revealed that the invasive impairment in AFP treatment was obviously lower than that in incision-thread-drawing group.

\section{Anus function}

The anus function was evaluated by preoperative and postoperative Wexner score between the two groups. The data presented that the anal function score was 0 (range $0 \sim 0$ ) in AFP group and 4 (range $0 \sim 16$ ) in incision-thread-drawing group. Hence, there were notably changes in anus function between the two groups $(\mathrm{Z}=-4.88, \mathrm{P}<0.001)$. Furthermore, none of patients with AFP treatment didn't manifested anal discharge, anus itching and anus damp, but patients with incision-thread-drawing had the above symptoms. Thereby, these data implied that it was better to maintain the anus function in anal fistula with AFP operation.

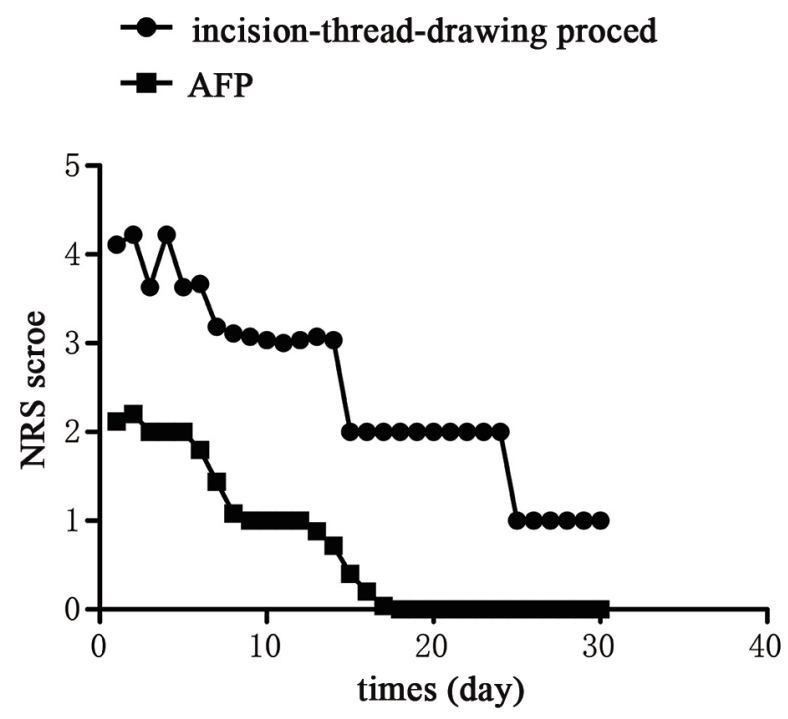

Figure 1. Comparisons of NRS score between AFP and incisionthread-drawing operations.

\section{Postoperative pain}

The evaluation of the preoperative pain showed that there were no significant differences in NRS score between AFP and incision-thread-drawing operations. Moreover, the NRS scores in patients with AFP operation were gradually decreased over time except for the first day when parecoxib sodium was given to relieve pain once a day (Figure 1). Nevertheless, the NRS scores in patients with incision-thread-drawing operation were also declined with time except for the first and third day when ketorolac tromethamine or parecoxib sodium was used to relieve pain twice a day (Figure 1). At other days after surgery, the patients of the two groups were commonly administrated with celebrex or didofence sodium enteric-coated tablets to alleviate pain (Figure 1). Overall, the NRS scores at every day of one month after AFP surgery were significantly lower than those after incision-thread-drawing surgery $(\mathrm{P}<0.001)$.

\section{The absorption of $A F P$}

As shown in Figure 2A, it was found that a cable-like high signal was located in bottom left of anus which represented anal fistula before AFP surgery, while in Figure 2B, it was discovered that the scar tissue had been formed in original fistula location and anal fistula has also been partially absorbed after 6 months of AFP surgery.

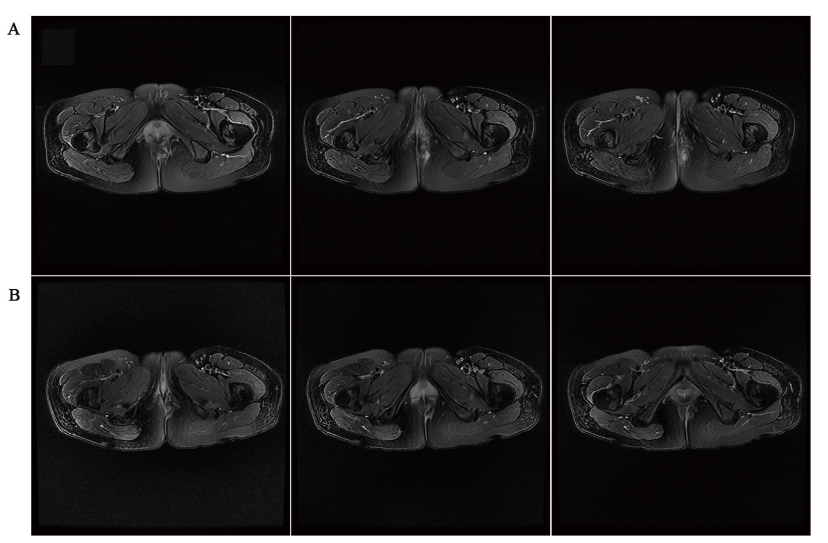

Figure 2. MRI scanning pictures of anal fistula. (A) Anal fistula before AFP surgery. (B) Anal fistula after 6 months of AFP surgery.

Table 1. The clinical data of two group's patients.

\begin{tabular}{|c|c|c|}
\hline Clinical data & AFP ( 25 cases) & Incision-thread-drawing (27 cases) \\
\hline Age & $36.56 \pm 10.56$ & $37.48 \pm 14.29$ \\
\hline Male/female & $22 / 3$ & $25 / 2$ \\
\hline Duration (month) & 4 & 6 \\
\hline Median & $(1-12)$ & $(0.23-60)$ \\
\hline \multicolumn{3}{|l|}{ External fistula (n) } \\
\hline Front & 5 & $6^{*}$ \\
\hline Lateral & 9 & $18^{*}$ \\
\hline Back & 11 & $3^{*}$ \\
\hline $\begin{array}{l}\text { Length of the fistula } \\
(\mathrm{cm})\end{array}$ & $7.14 \pm 1.91$ & $7.18 \pm 2.18$ \\
\hline$\leq 7$ & 15 & 16 \\
\hline$>7$ & 10 & 11 \\
\hline \multicolumn{3}{|l|}{ Types of fistula (\%) } \\
\hline $\begin{array}{l}\text { High anal fistula } \\
\text { (complex, simple) }\end{array}$ & $(9,14)$ & $(10,10)$ \\
\hline $\begin{array}{l}\text { Low anal fistula } \\
\text { (complex, simple) }\end{array}$ & $(0,2)$ & $(2,5)$ \\
\hline
\end{tabular}




\begin{tabular}{lcc}
\hline $\begin{array}{l}\text { Number of external } \\
\text { orifice }\end{array}$ & 21 & 20 \\
\hline $\mathrm{n}=1$ & 4 & 7 \\
\hline $\mathrm{n}>1$ & 21 & $16^{*}$ \\
\hline Number of fistula & & $11^{*}$ \\
\hline $\mathrm{n}=1$ & 4 & \\
\hline $\mathrm{n}>1$ & & \\
\hline${ }^{*} \mathrm{P}<0.05$ vs. AFP. & & \\
\hline
\end{tabular}

Table 2. Two groups of postoperative evaluation index contrast.

\begin{tabular}{lll}
\hline Contrast indicators & AFP & Incision-thread-drawing \\
\hline Number of healing & 19 & 25 \\
\hline Heal & 6 & 2 \\
\hline Inefficient & $31.96 \pm 2.54$ & $39.44 \pm 12.42^{*}$ \\
\hline Healing time (d) & 33 & $40^{*}$ \\
\hline Median healing time (d) & $(26-36)$ & $(21-68)$ \\
\hline Range (d) & $0.33 \pm 0.12$ & $3.06 \pm 1.62^{* *}$ \\
\hline Scar area (cm $\left.{ }^{2}\right)$ & 0 & $4^{* *}$ \\
\hline Anus function (median) & 0 & $(0-16)$ \\
\hline Range & $(0-0)$ & \\
\hline After the wound healing & & $2^{* *}$ \\
\hline Anal discharge & 0 & $5^{* *}$ \\
\hline Anus itching & 0 & $4^{* *}$ \\
\hline Anus damp & 0 & \\
\hline${ }^{*} \mathrm{P}<0.05$ vs. APF and ${ }^{* *} \mathrm{P}<0.01$ vs. APF. & \\
\hline
\end{tabular}

\section{Discussion}

Although anal fistula is a common disease in anorectal, the treatment methods of anal fistulas have remained a perplexing medical challenge because their lower healing rate, higher recurrence rate and serious faecal continence impairments [11]. Therefore, a large amount of anorectal surgeons constantly sought for the new technology to improve the clinical outcomes of anal fistula treatments over the past decades. AFP, a natural biomaterial harvested from porcine small intestine, could be fabricated into a biomedical product of various shapes and thickness [12]. It has been reported that AFP might be considered as a new therapeutic technology for anal fistula treatments due to it is an absorbable material and has an inherent resistance to infection [13]. For example, Champagne et al. demonstrated that cryptoglandular fistulas patients obtained a successful closure with an overall success rate of $83 \%$ by using AFP made of cell-free matrix freeze-dried pig small intestinal submucosa [14]; many surgeons have used AFP to treat Crohn's disease patients [15] and Acquired Immune Deficiency Syndrome (AIDS) patients [16] with anal fistula and obtained a better curative effect. Hence, AFP may be a promising application in clinic for anal fistula treatments. In present study, our results showed that there were no significant differences in healing rates between AFP treatments and incision-thread-drawing treatments. It is well known that preconditions of a successful anal fistula surgery include the internal orifice position, the length of the anal fistula and the depth of the fistula [17]. When the internal orifice position is higher than the dentate line, the length and depth of the anal fistula would be longer, thereby the successful rate of its surgery might be higher. However, these indicators were not remarkably difference in the two groups, which might be lead to no differences in the healing rates stated above. Additionally, the total and median healing time of AFP operation was both shorter than these of incision-threaddrawing operation. Moreover, a meta-analysis revealed that AFP surgery could obviously reduce the healing rate of patients with a complex anal fistula from $24.5 \pm 5.5 \mathrm{~d}$ to $7.5 \pm$ $3.5 \mathrm{~d}$ [18]. Thus, these findings suggested that AFP might be an effective procedure for anal fistula patients.

Postoperative complications, including bleeding, anus itching, auns damp, urinary retention, anal discharge, pain and faecal impaction, etc., are also important indexes to further evaluate the clinical efficacy of these surgeries [19]. Our data verified that no obvious complications occurred in AFP group, but some patients with incision-thread-drawing operations were induced the complications, such as anal discharge, anus itching and anus damp. Hence, these data indicated that AFP treatments might have a better prognosis for anal fistula patients. In addition, there currently were no reports on the comparative assessment of postoperative pain between AFP operation and incision-thread-drawing operation, thereby the degree and the duration of postoperative pain for the first time were also assessed by NRS scores in the two groups in this study. The results uncovered that the NRS scores in AFP group were notably less than these in incision-thread-drawing group at every day of the first month after operations, implying that AFP treatment could further alleviate postoperative pain as compared to incision-thread-drawing treatment. Furthermore, during the incision-thread-drawing procedure, surgeons should carefully cut the sphincter, made a long fusiform incision at the external orifice and remove the damaged tissues around fistulas $[4,20]$. Nevertheless, the above processes were not done in AFP procedure. Therefore, these results turned out that AFP surgery possesses obvious advantages in reducing invasive injures and postoperative pain. However, the long-term clinical efficacy of AFP is still unknown in this study because it lacked of sufficient patients with AFP treatments and the follow-up visit only persisted 3 months. Furthermore, accumulating evidence demonstrated that the recurrence rate in complexity anal fistula patients following AFP surgery significantly elevated concomitant with the prolongation of follow-up time $[11,18]$. Thus, it is necessary to further confirm our results that AFP is a good choice for treating anal fistula patients by increasing the number of patient with AFP operation and prolonging the length of follow-up time.

Taken together, we revealed that in comparison with incisionthread-drawing operation, AFP had an easier operation, made a 
smaller surgical trauma, shortened the healing time and avoided the anus function impairments. However, based on these results analysed by a retrospective analysis, in-depth investigations need to further confirm the accuracy of our conclusion by larger samples and multi-centered randomized controlled trials, etc. in the future.

\section{Acknowledgement}

None

\section{Funding}

None

\section{Competing Financial Interests Statement}

The authors declare no conflicts of interest.

\section{Ethic Approval}

This study was performed after the approval of the Medical Research Ethics Committee and has therefore been performed in accordance with the ethical standards laid down in an appropriate version of the Declaration of Helsinki (as revised in Brazil 2013).

\section{Informed Consent}

Written informed consents obtained from all participants included in the study.

\section{References}

1. Lewis R, Lunniss PJ, Hammond TM. Novel biological strategies in the management of anal fistula. Colorectal Dis 2012; 14: 1445-1455.

2. Chung W, Kazemi P, Ko D, Sun C, Brown CJ, Raval M, Phang T. Anal fistula plug and fibrin glue versus conventional treatment in repair of complex anal fistulas. Am J Surg 2009; 197: 604-608.

3. Malik AI, Nelson RL. Surgical management of anal fistulae: a systematic review. Colorectal Dis 2008; 10: 420-430.

4. Song KH. New techniques for treating an anal fistula. J Korean Soc Coloproctol 2012; 28: 7-12.

5. Limura E, Giordano P. Modern management of anal fistula. World J Gastroenterol 2015; 21: 12-20.

6. Steele SR, Kumar R, Feingold DL, Rafferty JL, Buie WD, Standards Practice Task Force of the American Society of Colon and Rectal Surgeons. Practice parameters for the management of perianal abscess and fistula-in-ano. Dis Colon Rectum 2011; 54: 1465-1474.

7. Kockerling F, Alam NN, Narang SK, Daniels IR, Smart NJ. Treatment of fistula-in-ano with fistula plug-a review under special consideration of the technique. Front Surg 2015; 2: 55.
8. Cadeddu F, Salis F, Lisi G, Ciangola , Milito G. Complex anal fistula remains a challenge for colorectal surgeon. Int J Colorectal Dis 2015; 30: 595-603.

9. Johnson EK, Gaw JU, Armstrong DN. Efficacy of anal fistula plug vs. fibrin glue in closure of anorectal fistulas. Dis Colon Rectum 2006; 49: 371-376.

10. Ratto C, Litta F, Donisi L and Parello A. Prospective evaluation of a new device for the treatment of anal fistulas. World J Gastroenterol 2016; 22: 6936-6943.

11. Akiba RT, Rodrigues FG, da Silva G. Management of complex perineal fistula disease. Clin Colon Rectal Surg 2016; 29: 92-100.

12. Saba RB, Tizmaghz A, Ajeka S, Karami M. Treating anal fistula with the anal fistula plug: case series report of 12 patients. Electron Phys 2016; 8: 2304-2307.

13. Herold A, Ommer A, Furst A, Pakravan F, Hahnloser D, Strittmatter B, Schiedeck T, Hetzer F, Aigner F, Berg E, Roblick M, Bussen D, Joos A. Results of the gore bio-a fistula plug implantation in the treatment of anal fistula: a multicentre study. Tech Coloproctol 2016; 20: 585-590.

14. Champagne BJ, OConnor LM, Ferguson M, Orangio GR, Schertzer ME, Armstrong DN. Efficacy of anal fistula plug in closure of cryptoglandular fistulas: long-term follow-up. Dis Colon Rectum 2006; 49: 1817-1821.

15. OConnor L, Champagne BJ, Ferguson MA, Orangio GR, Schertzer ME, Armstrong DN. Efficacy of anal fistula plug in closure of Crohns anorectal fistulas. Dis Colon Rectum 2006; 49: 1569-1573.

16. Uba AF, Chirdan LB, Ardill W, Ramyil VM, Kidmas AT. Acquired rectal fistula in human immunodeficiency viruspositive children: a causal or casual relationship? Pediatr Surg Int 2004; 20: 898-901.

17. Fogel S. Surgical failures: is it the surgeon or the patient? The all too often missed diagnosis of Ehlers-Danlos syndrome. Am Surg 2013; 79: 608-613.

18. Leng Q, Jin HY. Anal fistula plug vs mucosa advancement flap in complex fistula-in-ano: a meta-analysis. World J Gastrointest Surg 2012; 4: 256-261.

19. Ommer A. Management of complications of fissure and fistula surgery. Chirurg 2015; 86: 734-740.

20. Balciscueta Z, Uribe N, Balciscueta I, Andreu-Ballester JC, Garcia-Granero E. Rectal advancement flap for the treatment of complex cryptoglandular anal fistulas: a systematic review and meta-analysis. Int $\mathrm{J}$ Colorectal Dis 2017; 32: 599-609.

\section{*Correspondence to}

Chenguo Zheng

Department of Anorectal Surgery

The Second Affiliated Hospital and Yuying Children's Hospital of Wenzhou Medical University

PR China 